\title{
Communicating vein between the right external and internal jugular veins: a case report
}

\author{
Eleni Patera (D), Abduelmenem Alashkham (D) \\ Anatomy, School of Biomedical Sciences, Edinburgh Medical School, University of Edinburgh, Edinburgh, UK
}

\begin{abstract}
The external jugular vein is a superficial vein that has a relatively diagonal to vertical course in the neck region and runs superficial to the sternocleidomastoid muscle. This vein is formed by the union of the posterior division of the retromandibular vein with the posterior auricular vein and it is responsible for draining most of the scalp and face as well. Sound knowledge of variations of the external jugular veins and the internal jugular veins, is important as these veins are used or targeted in specific medical procedures such as external jugular vein cannulation or radical neck dissection, respectively. During routine postgraduate dissection of the neck region in a 58-year-old female cadaver, the right external jugular vein was seen communicating with the right internal jugular vein via a communicating vein. The communicating vein was located approximately at the lower border of the thyroid cartilage and the upper border of the cricoid cartilage. A thorough understanding of anatomical variations is important in various medical disciplines and more specifically to anatomists, radiologists, and surgeons. This case report does not solely aim to increase awareness regarding variations of the jugular veins that can be possibly encountered during a neck endovascular procedure, but also contribute to the identification of the prevalence rate of this variation.
\end{abstract}

Keywords: anatomical variation; anatomy; communicating vein; jugular veins

Anatomy 2021;15(1):84-87 C2021 Turkish Society of Anatomy and Clinical Anatomy (TSACA)

\section{Introduction}

The external jugular vein is a superficial vein in the lateral cervical region which is responsible for draining most of the scalp and side of face. It begins near the angle of the mandible, inferior to the auricle and is formed by the union of the posterior division of the retromandibular vein with the posterior auricular vein. ${ }^{[1]}$ The external jugular vein descends from the angle of the mandible to the middle of the clavicle. Through its course the external jugular vein crosses the sternocleidomastoid muscle obliquely and superficial to it. The external jugular vein is deep to the platysma and eventually enters the anteroinferior part of the lateral cervical region. The external jugular vein pierces the investing layer of the deep cervical fascia at the posterior border of the sternocleidomastoid muscle, descends to the inferior part of the lateral cervical region and terminates in the subcla- vian vein lateral or anterior to the scalenus anterior muscle. $^{[1,2]}$

The internal jugular vein receives blood from the brain, scalp, superficial parts of the face, and neck. The internal jugular vein is a continuation of the sigmoid sinus and it begins at the cranial base in the posterior compartment of the jugular foramen from which it passes through and descends in the carotid sheath where it unites with the subclavian vein posterior to the sternal end of the clavicle to drain into the brachiocephalic vein. ${ }^{[1,2]}$

Awareness of jugular veins variations is significant during the conduction of endovascular procedures taking place in the neck region. The aim of this study was to report an anatomical variation of the right jugular veins that is different from the anatomical variations of the jugular veins that have been currently described in the literature and to contribute into the identification of the rate of which this anatomical variation is encountered.

This study has been presented as a poster at the BACA Winter Conference at New Castle University, United Kingdom in December 17, 2019. 


\section{Case Report}

This study was conducted in Anatomy, University of Edinburgh on five formalin fixed adult human cadavers aging between $58-101$ years of which 2 were male and 3 were female. A full dissection of the thorax and neck regions was performed. The body was in the supine position and the neck was rested on a rubber neck rest allowing slight head extension and access to the neck structures. The platysma muscle and the sternal and clavicular heads of the sternocleidomastoid muscle were detached from their origin. The sternohyoid, sternothyroid and omohyoid muscles were removed from their origin and reflected upwards. Sharp dissection was performed by using a $15 \mathrm{~mm}$ scalpel to remove the carotid sheath of the deep cervical fascia that covered the carotid vessels and the internal jugular veins and to expose the jugular veins.

In a 58-year-old female cadaver, the right and left external jugular veins were normally formed by the union of the posterior division of the retromandibular vein and the posterior auricular vein. The right external jugular vein was seen communicating with the right internal jugular vein via a communicating vein (Figure 1). This variation occurred only in one out of the five cadavers. The communicating vein was posterior to the sternocleidomastoid muscle and was located approximately at the lower border of the thyroid cartilage and the upper border of the cricoid cartilage. The communicating vein was approximately $1.5 \mathrm{~cm}$ in length and its diameter was around 3.82 $\mathrm{mm}$. The right internal jugular vein was larger in diameter compared with the right external jugular vein. The right external and internal jugular veins drained normally into the right subclavian and right brachiocephalic vein, respectively. No variations were encountered in the jugular veins of the left side of the neck.

\section{Discussion}

Classical anatomical textbooks describe the external jugular vein being formed by the posterior division of the retromandibular vein and the posterior auricular vein. ${ }^{[1-3]}$ The internal jugular vein is often described to be formed by the union of the sigmoid and inferior petrosal venous sinuses. Classically, there is one external and one internal jugular vein on each side of the neck and no communication between these veins.

In the current literature, variations of the jugular veins have been well reported. Paraskevas et al. ${ }^{[4]}$ reported a rare case where three external jugular veins were found to co-exist with two anterior jugular veins. In contrast, another case report reported absence of the left EJV. As the EJV was absent, the posterior division of the retromandibular vein drained directly into the IJV. ${ }^{[5]}$ Kayiran et al. ${ }^{[6]}$ reported absence of the right IJV which was identified primarily by ultrasonography, CT, and MRI scans, after the patient presented with a painless cervical mass on the lateral side of the neck. The left IJV was observed to be significantly thicker. The authors attributed this case as a congenital agenesis event.

In the literature, there are currently three published case reports that describe communication between the external and internal jugular veins. Uemura et al., ${ }^{[7]}$ published a rare case report of a right partial and double IJVs. Initially, authors reported in their abstract that the right IJV was found to bifurcate into a medial and a lateral branch at the middle of the fifth cervical vertebral body

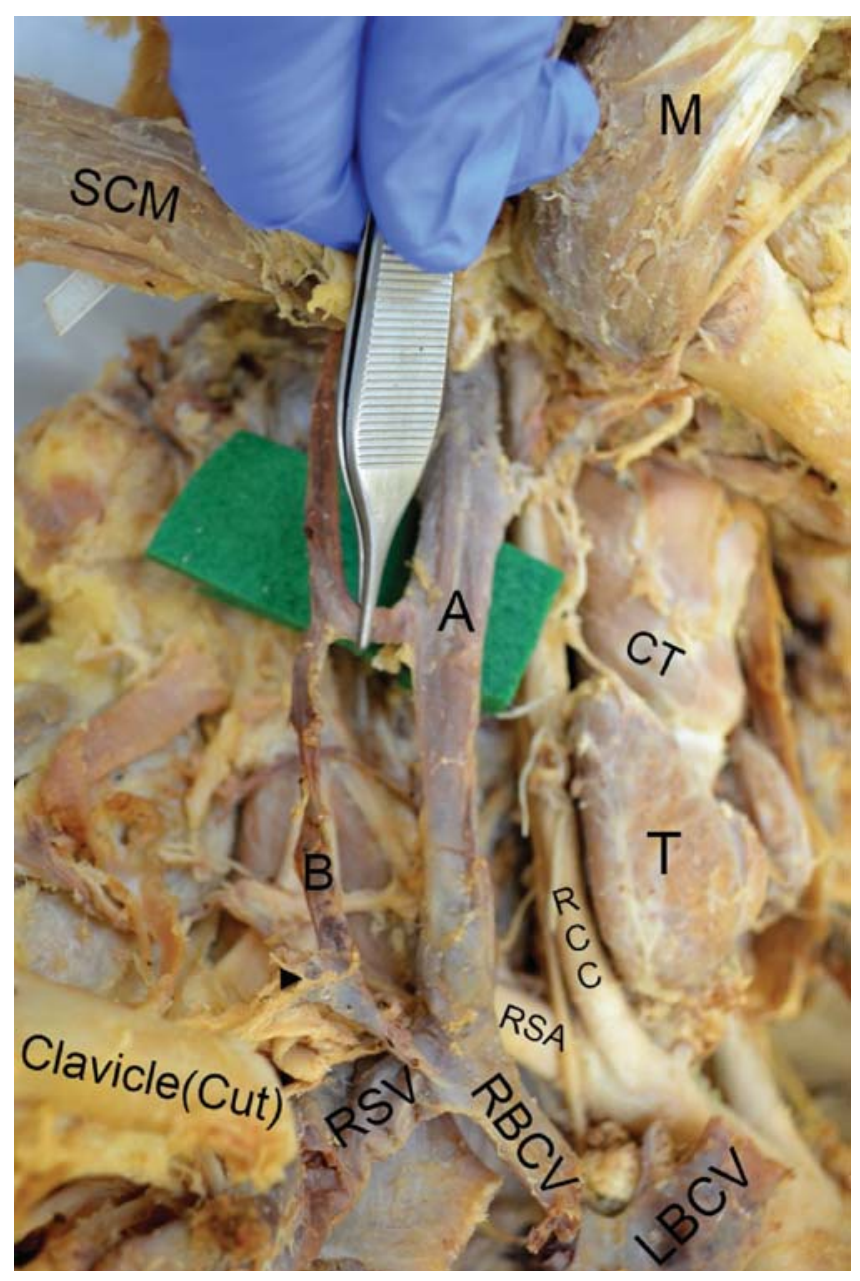

Figure 1. Lateral view of the right side of the neck region showing the variation of the jugular veins. The communicating vein held by the forceps is found between the right internal jugular vein $(A)$ and the right external jugular vein (B). CT: cricothyroid muscle; LBCV: left brachiocephalic vein; M: masseter; RBCV: right brachiocephalic vein; RCC: right common carotid artery; RSA: right subclavian artery; RSV: right subclavian vein; SCM: sternocleidomastoid muscle (reflected); T: thyroid gland. 
with both branches draining into the right subclavian vein. Subsequently in their results, authors mentioned that the lateral branch of the right IJV joined the right EJV whereas the medial branch of the right IJV drained directly into the right subclavian vein. The medial and lateral branches of the IJV ran parallel during their descending course in the neck. In addition, the authors reported that the EJV was formed by the confluence between the right superficial temporal and maxillary veins which then divided into a medial and lateral branch, at the level of the superior margin of the right SCM muscle. The medial and lateral branches of the right EJV joined again at the inferior border of the SCM and drained into the distal portion of the right EJV. The right EJV along with the lateral branch of the right IJV eventually drained into the right subclavian vein. Authors concluded that the medial branch of the right IJV was in fact the right IJV and that the lateral branch of the right IJV has in fact developed from the right EJV because the anterior jugular vein drained into it. Hence, they concluded that the lateral branch of the right EJV was a communicating branch between the right EJV and IJV. Overall, there is some ambiguity in this case report as authors primarily mentioned that the right IJV gave a medial and a lateral branch which they then concluded that it was instead a branch of the right EJV that communicated the right EJV and IJV. Further ambiguity toward our understanding over this anatomical variation is present, as the cadaveric images provided in this article do not allow the reader to have a clear view over the course of the veins described and their drainage pathway as well.

Lalwani et al. ${ }^{[8]}$ reported an abnormal communication between the EJV and the IJV. The authors reported that a venous channel arose from the EJV, traversing the anterior border of the sternocleidomastoid muscle ultimately descending to drain into the IJV. Authors failed to show an image of this venous channel emptying in the IJV as deep dissection to remove the SCM muscle to expose the IJV was not performed. No information was provided regarding the length of the venous channel that was found between the EJV and IJV. In contrast, results from our case report, report a communicating vein between the right EJV and IJV that was identified after performing deep neck dissection (Figure 1).

Another rare case report by Karapantzos et al..$^{[9]}$ reported an anastomosis between the right EJV and IJV. Authors mentioned that this shunt seemed to come off the right $\mathrm{EJV}$, passed through the sternocleidomastoid muscle eventually leading to the IJV where it drained into eventually allowing authors to conclude that this shunt was an extra EJV. The figure showing this anastomosis between the EJV and IJV hinders our understanding over this variation, as adjacent anatomical structures including the sternocleidomastoid muscle and thyroid cartilage were not indicated. Additionally, by looking at the figure, it seems that the shunt was posterior to the sternocleidomastoid muscle, it ran obliquely eventually draining into the left IJV and not the right IJV as authors reported.

In contrast to the aforementioned studies that reported a communication between the EJV and IJV, results from our case report, report a communicating vein between the right EJV and IJV that was posterior to the sternocleidomastoid muscle and was located approximately at the level of the upper border of the cricoid cartilage. Its length was approximately $1.5 \mathrm{~cm}$, its diameter was around $3.82 \mathrm{~mm}$ and its course was horizontal. The communicating vein between the right EJV and IJV described in our current case report, differs significantly from the communicating vein between the jugular veins that has been previously described in the literature by Uemura et al., ${ }^{[7]}$ Lalwani et al., ${ }^{[8]}$ and Karapantzos et al. ${ }^{[9]}$ Such anatomical variation has not been previously described in the current literature to the best of our knowledge.

Knowledge of variations of the jugular veins is indispensable prior the conduction of surgical endovascular procedures within the neck region including radical neck dissection surgeries. Radical neck dissection is a surgical oncology procedure that is being conducted for the management of metastatic nodal disease in the neck. ${ }^{[10]}$ As the neck is a region that is rich in lymph nodes, radical neck dissection is performed in individuals with certain types of head and neck cancer. ${ }^{[11]}$ In the past, Conley described radical neck dissection as the most effective surgical procedure for controlling suspected or gross metastasis in the neck region via the removal of lymph nodes in the neck. ${ }^{[12]}$ Despite this, Harish highlighted that when extracapsular spread or perineural invasion due to lymph node metastasis is present, radical neck dissection is not adequate for the removal of the tumour thus, the use of radiation in addition to the radical neck dissection might be necessary for specific patients. ${ }^{[13]}$

In radical neck dissection, the internal jugular vein undergoes removal along both the sternocleidomastoid muscle and the spinal accessory nerve. In modified radical neck dissection type I, the internal jugular vein and the sternocleidomastoid muscle undergo removal. In modified radical neck dissection type II, the internal jugular vein is preserved. ${ }^{[10]}$

Knowledge of variations of the jugular veins such as a communicating vein between the external and internal 
jugular veins is indispensable prior the conduction of radical neck dissection and modified radical neck dissection type I as the EJV is ligated prior the IJV. Therefore, unawareness of such variation can eventually lead to excessive bleeding.

\section{Conclusion}

Overall, a thorough understanding of variations of the jugular veins is indispensable prior the conduction of surgical endovascular procedures within the neck region. Additionally, the documentation of anatomical variations contributes to the identification of the actual prevalence rate of specific anatomical variations within a body region. Once, an anatomical variation has been reported in the literature to a great extent by numerous studies, then, it can be included as a frequently encountered anatomical variation in the anatomical variation section in anatomical textbooks.

\section{Acknowledgments}

The authors would like to express their sincere gratitude to the donors and their families. Special thanks to the Anatomy department at the University of Edinburgh.

\section{Conflict of Interest}

None.

\section{Author Contributions}

EP: Dissection, data collection, image acquisition, analysis and writing up; AA: Analysis, editing and proofreading.

\section{Ethics Approval}

The specimens were obtained from the Centre for Anatomy, which is regulated by the Anatomy Scotland Act (2006).

\section{Funding}

None.

\section{References}

1. Moore KL, Dalley AF, Agur AMR, editors. Clinically oriented anatomy clinical anatomy. 7th ed. Philadelphia (PA): Wolters Kluwer; 2017. p. 995-1004.

2. Standring S, editor. Gray's anatomy: The anatomical basis of clinical practice. 41st ed. London: Churchill Livingstone Elsevier; 2015. p. 406-14.

3. Wineski L, editor. Snell's clinical anatomy by regions. 10th ed. London: Wolters Kluwer; 2018. p. 1617-46.

4. Paraskevas G, Natsis K, Ioannidis O, Kitsoulis P, Anastasopoulos N, Spyridakis I. Multiple variations of the superficial jugular veins: case report and clinical relevance. Acta Medica (Hradec Kralove) 2014;57:34-7.

5. Abhinitha P, Rao MKG, Kumar N, Nayak SB, Ravindra SS, Aithal PA. Absence of the external jugular vein and an abnormal drainage pattern in the veins of the neck. OA Anatomy 2013;1:1-3.

6. Kayiran O, Calli C, Emre A, Soy FK. Congenital agenesis of the internal jugular vein: an extremely rare anomaly. Case Rep Surg 2015;2015:637067.

7. Uemura M, Takemura A, Tamada Y, Toda I, Ike H, Suwa F. A case of right partial and double internal jugular veins. Anat Sci Int 2006;81:65-8.

8. Lalwani R, Rana K, Das S, Khan R. Communication of the external and internal jugular veins: a case report. International Journal of Morphology 2006;24:721-2.

9. Karapantzos I, Zarogoulidis P, Charalampidis C, Karapantzou C, Kioumis I, Tsakiridis K, Mpakas A, Sachpekidis N, Organtzis J, Porpodis K, Zarogoulidis K, Pitsiou G, Zissimopoulos A, Kosmidis C, Fouka E, Demetriou T. A rare case of anastomosis between the external and internal jugular veins. Int Med Case Rep J 2016;9:73-5.

10. Gogna S, Gupta N. Cancer, neck resection and dissection. In: StatPearls [Internet]. Treasure Island (FL): StatPearls Publishing; 2021. Available from: https://www.ncbi.nlm.nih.gov/books/NBK536998/

11. Koroulakis A, Jamal Z, Agarwal M. Anatomy, head and neck, lymph nodes. In: StatPearls [Internet]. Treasure Island (FL): StatPearls Publishing; 2021. Available from: https://www.ncbi.nlm.nih.gov/ books/NBK513317/

12. Conley J. Radical neck dissection. Laryngoscope 1975;85:1344-52.

13. Harish K. Neck dissections: radical to conservative. World J Surg Oncol 2005;3:21.
ORCID ID:

E. Patera 0000-0001-6154-4069; A. Alashkham 0000-0003-2959-5039
Correspondence to: Abduelmenem Alashkham, $\mathrm{PhD}, \mathrm{MSc}, \mathrm{MBBCh}$ University of Edinburgh, Old Medical School, Doorway 3, Teviot Place, Edinburgh, EH8 9AG, UK

Phone: +44 1316502924

e-mail: abduelmenem.alashkham@ed.ac.uk

Conflict of interest statement: No conflicts declared.

This is an open access article distributed under the terms of the Creative Commons Attribution-NonCommercial-NoDerivs 4.0 Unported (CC BY-NCND4.0) Licence (http://creativecommons.org/licenses/by-nc-nd/4.0/) which permits unrestricted noncommercial use, distribution, and reproduction in any medium, provided the original work is properly cited. How to cite this article: Patera E, Alashkham A. Communicating vein between the right external and internal jugular veins: a case report. Anatomy 2021;15(1):84-87. 